\title{
Candidate microRNA biomarkers of pancreatic ductal adenocarcinoma: meta-analysis, experimental validation and clinical significance
}

\author{
Ming-Zhe Ma ${ }^{1 \dagger}$, Xiang Kong ${ }^{2,3 \dagger}$, Ming-Zhe Weng ${ }^{1 \dagger}$, Kun Cheng $^{4}$, Wei Gong ${ }^{1 *}$, Zhi-Wei Quan ${ }^{1 *}$ \\ and Cheng-Hong Peng ${ }^{4}$
}

\begin{abstract}
Background: The diagnostic and prognostic value of microRNA (miRNA) expression aberrations in pancreatic ductal adenocarcinoma (PDAC) has been studied extensively in recent years. However, differences in measurement platforms and lab protocols as well as small sample sizes can render gene expression levels incomparable.

Methods: A comprehensive meta-review of published studies in PDAC that compared the miRNA expression profiles of PDAC tissues and paired neighbouring noncancerous pancreatic tissues was performed to determine candidate miRNA biomarkers for PDAC. Both a miRNA vote-counting strategy and a recently published Robust Rank Aggregation method were employed. In this review, a total of 538 tumour and 206 noncancerous control samples were included.

Results: We identified a statistically significant miRNA meta-signature of seven up- and three down-regulated miRNAs. The experimental validation results showed that the miRNA expression levels were in accordance with the meta-signature. The results from the vote-counting strategy were consistent with those from the Robust Rank Aggregation method. The experimental validation confirmed that the statistically unique profiles identified by the meta-review approach could discriminate PDAC tissues from paired nonmalignant pancreatic tissues. In a cohort of 70 patients, the high expression of miR-21 ( $p=0.018, \mathrm{HR}=2.610 ; 95 \% \mathrm{Cl}=1.179-5.777)$ and miR-31 ( $p=0.039, \mathrm{HR}=2.735$; $95 \% \mathrm{Cl}=1.317-6.426)$, the low expression of miR-375 ( $p=0.022, \mathrm{HR}=2.337 ; 95 \% \mathrm{Cl}=1.431-5.066)$ were associated with poor overall survival following resection, independent of clinical covariates.
\end{abstract}

Conclusions: The identified miRNAs may be used to develop a panel of diagnostic and prognostic biomarkers for PDAC with sufficient sensitivity and specificity for use in a clinical setting.

Keywords: microRNA, Meta-analysis, Pancreatic cancer, Biomarker

\section{Introduction}

Pancreatic ductal adenocarcinoma (PDAC) is one of the most aggressive of all malignancies [1]. Less than $20 \%$ of PDAC patients present with localised, potentially curable tumours. The overall 5 -year survival rate is $<5 \%$. Because the chemotherapeutic options prolong life only minimally, the current PDAC mortality is nearly identical to its incidence [2]. Extensive studies have been performed to

\footnotetext{
*Correspondence: gongweius@hotmail.com; zhiwquan@163.com ${ }^{\dagger}$ Equal contributors

'Department of General Surgery, Xinhua Hospital, Shanghai Jiaotong University School of Medicine, 1665 Kongjiang Road, Shanghai 200092, People's Republic of China

Full list of author information is available at the end of the article
}

identify biomarkers for this disease. At the messenger RNA (mRNA) level, quite a few, including some very specific molecular variations have been found in cancerous tissues [3]. MicroRNAs (miRNAs), a class of short non-coding RNA molecules that range in size from 19 to 25 nucleotides, have been proposed as promising biomarkers of early cancer detection and accurate prognosis as well as targets for more efficient treatment [4,5]. MiRNAs play important roles in regulating the translation of many genes and the degradation of mRNAs through base pairing to partially complementary sites, predominately in the $3^{\prime}$ untranslated region [6,7]. Several studies have implicated miRNAs in the regulation of

\section{Ciomed Central}


tumour biology [8-10]. Model biomarkers should be easily quantifiable and associate strongly with clinical outcome, and miRNAs may match these criteria.

High-throughput technologies have been employed to identify differences in miRNA expression levels between normal and cancerous tissues. These studies have the potential to identify dozens or hundreds of differentially expressed miRNAs, although only a small fraction of them may be of actual clinical utility as diagnostic/prognostic biomarkers. Finding a meaningful way in which to combine different data sources is often a non-trivial task. Differences in measurement platforms and lab protocols as well as small sample sizes can render gene expression levels incomparable. Hence, it may be better to analyse datasets separately and then aggregate the resulting gene lists. This strategy has been applied to identify gene coexpression networks [11] and to define more robust sets of cancer-related genes [12,13] and miRNAs $[14,15]$.

In the meta-review approach, the results of several individual studies are combined to increase statistical power and subsequently resolve any inconsistencies or discrepancies among different profiling studies. In this study, we applied two meta-review approaches: the wellknown vote-counting strategy $[12,13]$, which is based on the number of studies reporting a gene as being consistently expressed and then further ranking these genes with respect to total sample size and average fold-change, and the recently published Robust Rank Aggregation method $[16,17]$. Pathway analysis was then performed to identify the physiological impact of miRNA deregulation in PDAC progression. Moreover, we further validated the most up-regulated and down-regulated miRNAs from the meta-review in a clinical setting. The expression levels of a subset of candidate miRNAs were assessed by quantitative real-time polymerase chain reaction (qRT-PCR). With the validation of candidate miRNAs, we selected the most promising miRNAs based on factors such as fold-change to explore their potential effects on the survival of PDAC patients after surgical resection.

\section{Materials and methods}

\section{Selection of studies and datasets}

The Scopus database (www.scopus.com) was searched for PADC miRNA expression profiling studies using search term TITLE-ABS-KEY [(mirna* OR microrna* OR mir-* OR mir) AND profil* AND (pancreas* cancer OR pancreatic carcinoma OR pancreas" neoplas" OR pancreatic tumo* OR carcinoma of pancreas* OR cancer of pancreas*)]. The same strategy was also applied to searches of the Gene Expression Omnibus (GEO; www.ncbi.nlm.nih.gov/geo/), ArrayExpress (http://www.ebi.ac.uk/arrayexpress/), and PubMed (www.ncbi.nlm.nih.gov/pubmed). The last search was performed on May 11, 2013. The titles and abstracts of the articles were screened, and the full text of the articles of interest was evaluated. We included only original experimental articles that were published in English and that compared the expression of miRNAs in PDAC tissue and noncancerous pancreatic tissue in humans. Articles were excluded based on the following criteria: (i) review articles, case reports or letters; (ii) non-English articles; (iii) studies of individual preselected candidate miRNAs; (iv) studies that used RTPCR for initial selection (the reasons for this exclusion criterion are explained in the Discussion section); (v) studies using cell lines or serum from PDAC patients; (vi) studies that did not use a miRNA microarray platform; (vii) studies profiling different histological subtypes; (viii) studies that did not include noncancerous tissue.

\section{Data extraction}

Two investigators (MM and XK) independently evaluated and extracted the data using standard protocols, and all discrepancies were resolved by a third investigator $(\mathrm{MW})$. From the full text and corresponding supplemental information, the following eligibility items were collected and recorded for each study: author, region, period, selection and characteristics of the recruited PDAC patients, platform of miRNA expression profiling, and the list of up- and down-regulated miRNAs and their corresponding fold-changes. When the gene list was not available, the authors were contacted directly. All miRNA names were standardised according to miRBase version 20 .

\section{Data processing}

\section{Vote-counting strategy}

The miRNAs were ranked according to their importance as follows: (i) number of comparisons in agreement (i.e., listing the same miRNAs as having a consistent direction of change and being differentially expressed, respectively); (ii) total number of samples for comparison in agreement; (iii) average fold-changes reported for comparisons in agreement. Total sample size was considered more important than average fold-change because many studies did not report a fold-change. Furthermore, the average fold-change was based solely on the subset of studies for which a fold change value was available.

\section{Robust rank aggregation method}

The list of extracted miRNAs was ranked based on their associated $p$-values (less than 0.05 was considered significant) when their fold-changes were not reported. All of the protocols for the Robust Rank Aggregation method are free to download at the comprehensive R Archive Network website (http://cran. r-project.org/). Details can be found in the package documentation (http://cran.r-project.org/web/packages/ RobustRankAggreg/RobustRankAggreg.pdf). This method 
assigns a $p$-value to each element in the aggregated list, which indicates how much better it is ranked compared with a null model, expecting random ordering. To assess the stability of the acquired $p$-values, leave-one-out crossvalidation was applied in the Robust Rank Aggregation algorithm. This analysis was repeated 10,000 times, and each time, one random gene list was left out of the analysis. The $p$-values acquired from each round for each miRNA were then averaged.

\section{MiRNA target prediction and enrichment analysis}

The mRNA targets of the miRNA genes were predicted using TargetScan (http://www.targetscan.org/), miRDB (http://mirdb.org/miRDB/), and miRANDA (http://www. microrna.org/microrna/getGeneForm.do), as each algorithm determines target binding differently. We selected targets from the miRANDA/miSVR search with scores less than -1.25 for further analysis. Enrichment analyses for KEGG and Panther pathways and Gene Ontology terms were performed with the GeneCodis tool (http:// genecodis.dacya.ucm.es/). The potential targets of each miRNA were used as input.

\section{Ethics statement}

Ethical approval for this study was obtained from the Department of General Surgery of Ruijin Hospital at Shanghai Jiaotong University (Shanghai, China). All patients provided informed written consent for their tissues to be used for scientific research and to publish their case details.

\section{Sample collection}

Seventy-eight PDAC tissue samples and neighbouring noncancerous pancreatic tissue samples (collected postoperatively from September 2010 to August 2011) used in this study were obtained from the Department of General Surgery of Ruijin Hospital at Shanghai Jiaotong University (Shanghai, China). The specimens were obtained from patients undergoing PDAC resection with curative intent. All diagnoses were based on pathological and/or cytological evidence. The histological features of the specimens were evaluated by a senior pathologist according to the WHO (World Health Organization) classification criteria. The tissues were obtained before chemotherapy and radiation therapy. Upon removal of the surgical specimen, research personnel immediately

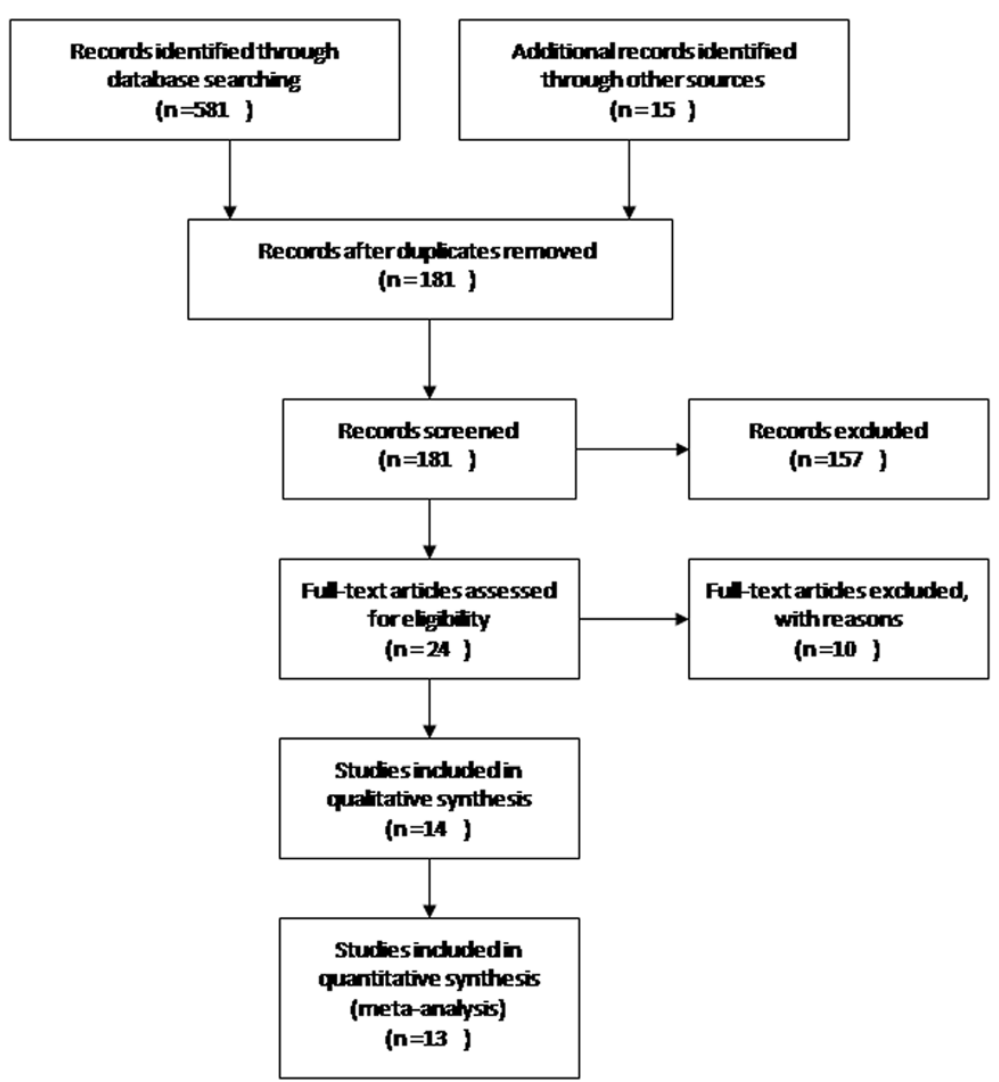

Figure 1 PRISMA 2009 flow chart. Only original experimental articles that were published in English and that analysed the differences in miRNA expression between PDAC tissue and noncancerous pancreatic tissue in humans were included. Articles were excluded if the studies did not use a miRNA microarray platform or if they profiled miRNAs in different histological subtypes. 
transported the tissue to the surgical pathology lab. Pathology faculty performed a gross analysis of the specimen and selected pancreatic tissues that appeared to be cancerous and pancreatic tissues that appeared to be normal for analysis. Each sample was immediately frozen in liquid nitrogen and stored at $-80^{\circ} \mathrm{C}$ prior to RNA isolation and qRT-PCR analysis. A second level of quality control was performed on the adjacent benign tissues. Histological slides were prepared from the section of frozen tissue that was directly adjacent to the tissue from which the RNA was isolated. These slides were examined by experienced pathologists to determine if the benign tissues contained any pancreatic tumour cells. Benign tissues that contained residual tumour tissues were not included in the study. Complete clinicopathological follow-up data of the PDAC patients from which the specimens were collected were available.

\section{Validation of the most up-regulated or down-regulated miRNAs using qRT-PCR}

Total RNA was isolated from the frozen tissue sample with TRIzol (Invitrogen) according to the manufacturer's instructions. First-strand complementary DNA (cDNA) was synthesised from $2 \mu \mathrm{g}$ of the total RNA using an oligo-dT primer and superscript II reverse transcriptase (Invitrogen). Then, quantification of the most up-regulated or down-regulated miRNAs was performed by qRT-PCR using SYBRR Premix Ex Taq (TakaRa). The U6 primers were obtained from TakaRa. PCR was performed in a real-time PCR system (BioRad) as follows: $95^{\circ} \mathrm{C}$ for $3 \mathrm{~min}$, followed by 35 cycles of $95^{\circ} \mathrm{C}$ for $5 \mathrm{sec}, 60^{\circ} \mathrm{C}$ for $20 \mathrm{sec}$ and $72^{\circ} \mathrm{C}$ for $30 \mathrm{sec}$, and then $94^{\circ} \mathrm{C}$ for $1 \mathrm{~min}$ and $60^{\circ} \mathrm{C}$ for $1 \mathrm{~min}$, with an increase of $0.5^{\circ} \mathrm{C}$ per cycle. The expression level values were normalised to those of the small nuclear RNA U6 as a control. Relative fold-changes of miRNA expression were calculated using the $\Delta \Delta \mathrm{CT}$ method, and the values were expressed as $2^{-\Delta \Delta C T}$. The primer sequences were as follows: U6, 5'-CTCGCTTCGGCAGCACA-3' (forward), 5' -AACGCTTCACGAATTTGCGT-3' (reverse); miR-155, 5' -cgGCGGTTAATGCTAATCGTG-3' (forward), 5'-GTGCAGGGTCCGAGGT-3' (reverse); miR100, 5' -GAATTCCCATACTGGTTGGCTCCCGC-3' (forward), 5'-CTCGAGACGAATTCAATCGAAATAT TC-3' (reverse); miR-21, 5' -ACACTCCAGCTGGGT AGCTTATCAGACTGA-3' (forward), 5' -TGGTGTCG TGGAGTCG-3' (reverse); miR-221, 5' -CCCAGCATT 'TCTGACTGTTG-3' (forward), 5' -TGTGAGACCATT TGGGTGAA-3' (reverse); miR-31, 5'-ACGCGGCAA GATGCTGGCA-3' (forward), 5'-CAGTGCTGGGTCC GAGTGA-3' (reverse); miR-143, 5'-CCTGGCCTGAG ATGAAGCAC-3' (forward), 5' -CAGTGCTGGGTCCG AGTGA-3' (reverse); miR-23a, 5'-CTTGAACTCCTG GCCTGAAG-3' (forward), 5' -GCCAAAGAAACACTC ACAGCT-3' (reverse); miR-217, 5'-GCGTACTGCATC AGGAACTGATTGGA-3' (forward), 5' -GGGCACACA AAGGCAACTTTTGT-3' (reverse); miR-148a, 5' -TC AGTGCACTACAGAACTTTGT-3' (forward), 5'-GC 'TGTCAACGATACGCTACGT-3' (reverse); miR-375, 5' -GAAGATCTTGAGGTACATCGCAGAGGCCAG-

3' (forward), 5' -CATGCCATGGGGGCCGGAGCGGA AGACCC-3' (reverse).

\section{Statistical analysis}

Kaplan-Meier survival analysis was used to analyse the association between postoperative survival and the miRNA expression level measured by qRT-PCR, and the resulting curves were divided into two classes (high and low expression in comparison to the mean level of miRNA expression as the threshold). Survival analysis was performed for each clinical covariate to assess their influence on outcome using a log-rank test. A multivariate Cox regression

Table 1 Eleven microarray-based miRNA expression profiling studies of human PDAC tissues

\begin{tabular}{cccccc}
\hline First author (reference) & Acronym & Region & Assay type & No. of probes & No. of samples (cancer/normal) \\
\hline AE Szafranska [21] & AE & USA & Custom microarray & 377 & $13(8 / 5)$ \\
Ada Piepoli [22] & AP & Italy & Affymetrix GeneChip array & 866 & NR (cancer=17) \\
Andrea S.Bauer [23] & AS & Germany & Geniom biochip miRNA homo sapiens & NR & $110(94 / 16)$ \\
Eun Joo Lee [24] & EJ & USA & Agilent Human miRNA Microarray & NR & $28(15 / 13)$ \\
Mark Bloomston [25] & MB & USA & Custom miRNA microarray & 1100 & $130(65 / 65), 65$ pairs \\
Nicolai A.Schultz [26] & NA & Denmark & TaqMan array human microRNA A+B Cards v2.0 & 664 & $188(160 / 28)$ \\
Nigel B.Jamieson [27] & NB & USA & Agilent Human miRNA Microarray (version 2.0) & 723 & $58(48 / 10)$ \\
Nicole C.Panarelli [28] & NC & USA & FlexmiR miRNA microarray & 328 & $27(17 / 10)$ \\
S Ali [29] & SA & USA & LC Science Houston microarray & NR & $44(29 / 15)$ \\
Shuyu Zhang [30] & SZ & China & Exiqon miRCURY LNA array & 1200 & $40(20 / 20), 20$ pairs \\
Yuichi Nagao [31] & YN & Japan & Toray 3D-Gene miRNA microarray & $>900$ & $79(65 / 24)$
\end{tabular}

Abbreviations: $N R$ not reported, pairs, cancerous and normal samples from the same patient. 
Table 2 Up-regulated miRNAs $(n=50)$ reported in at least two expression profiling studies

\begin{tabular}{|c|c|c|c|c|}
\hline miRNA name & Studies with the same direction (reference) & No. of tissue samples tested & Mean fold-change & Mean rank \\
\hline hsa-miR-155 & $A E, A P, A S, E J, M B, N B, N C, Y N$ & 329 & 4.98 & 12.62 \\
\hline hsa-miR-21 & $A E, M B, N A, N B, N C, S Z, Y N$ & 376 & 2.95 & 12.29 \\
\hline hsa-miR-100 & $A E, A S, E J, M B, N B, N C, Y N$ & 317 & 8.07 & 13.00 \\
\hline hsa-miR-221 & $A E, A P, A S, E J, M B, N B, N C$ & 264 & 6.71 & 11.42 \\
\hline hsa-miR-31 & $A E, A P, A S, N A, Y N$ & 344 & 5.44 & 10.00 \\
\hline hsa-miR-10a & $A E, A S, M B, N B, Y N$ & 280 & 2.50 & 14.60 \\
\hline hsa-miR-23a & $A E, A P, A S, M B, N B$ & 229 & 3.46 & 22.60 \\
\hline hsa-miR-143 & $A E, A P, M B, N B, Y N$ & 203 & 4.03 & 9.40 \\
\hline hsa-miR-222 & $A E, A S, M B, N B, Y N$ & 199 & 2.77 & 11.20 \\
\hline hsa-miR-210 & $A E, A S, M B, N A$ & 323 & 2.97 & 16.00 \\
\hline hsa-miR-125a-5P & $A E, A P, A S, M B$ & 184 & 2.98 & 22.50 \\
\hline hsa-miR-145 & $A E, A P, A S, N B$ & 167 & 3.75 & 9.75 \\
\hline hsa-miR-181a & $A S, M B, N B$ & 207 & 4.83 & 13.33 \\
\hline hsa-miR-199a-3p & $A P, A S, Y N$ & 176 & 3.59 & 9.33 \\
\hline hsa-miR-23b & $A S, A P, M B$ & 176 & 3.09 & 42.33 \\
\hline hsa-miR-181b & $A E, A S, M B$ & 167 & 2.71 & 14.67 \\
\hline hsa-miR-199b-3p & $A E, A S, N B$ & 159 & 3.83 & 14.33 \\
\hline hsa-miR-331-3p & $A P, A S, N B$ & 159 & 1.83 & 35.33 \\
\hline hsa-miR-150 & $A E, A S, N B$ & 150 & 3.73 & 6.67 \\
\hline hsa-let-7i & $A E, A S, N B$ & 150 & 2.47 & 17.33 \\
\hline hsa-miR-214 & $A E, A S, N B$ & 147 & 3.63 & 11.00 \\
\hline hsa-miR-1246 & $A P, A S, S A$ & 140 & 3.37 & 42.67 \\
\hline hsa-miR-223 & $A E, M B, N B$ & 121 & 3.71 & 6.67 \\
\hline hsa-miR-24 & $A E, A P, N B$ & 70 & 2.50 & 26.67 \\
\hline hsa-miR-584 & $A S, N A$ & 254 & 5.81 & 64.50 \\
\hline hsa-miR-886-5p & AS, NA & 254 & 3.26 & 38.50 \\
\hline hsa-miR-205 & $\mathrm{MB}, \mathrm{NA}$ & 225 & 11.04 & 12.50 \\
\hline hsa-miR-142-3p & $N A, N B$ & 208 & 4.17 & 23.50 \\
\hline hsa-miR-451 & NA, SA & 189 & 28.36 & 16.00 \\
\hline hsa-miR-939 & $A P, N A$ & 177 & 4.76 & 22.50 \\
\hline hsa-miR-196b & $A E, N A$ & 173 & 11.93 & 3.00 \\
\hline hsa-miR-99a & $A S, Y N$ & 159 & 2.07 & 60.00 \\
\hline hsa-miR-181c & $A S, M B$ & 159 & 4.49 & 9.50 \\
\hline hsa-miR-199a-5p & $A S, N B$ & 142 & 2.64 & 18.50 \\
\hline hsa-miR-505 & $A S, N B$ & 142 & 1.87 & 34.50 \\
\hline hsa-miR-342-3p & $A S, N B$ & 142 & 1.67 & 55.50 \\
\hline hsa-miR-140-3p & $A S, N B$ & 142 & 1.58 & 61.00 \\
\hline hsa-miR-34a & $A S, N B$ & 142 & 1.31 & 56.50 \\
\hline hsa-miR-92a & $A S, S A$ & 123 & 6.64 & 10.00 \\
\hline hsa-miR-320a & AS, SA & 123 & 2.05 & 28.50 \\
\hline hsa-let-7e & $A P, A S$ & 111 & 4.31 & 36.50 \\
\hline hsa-miR-92b & $A P, A S$ & 111 & 1.66 & 47.50 \\
\hline hsa-miR-224 & $A E, A S$ & 102 & 1.32 & 59.00 \\
\hline hsa-miR-99b & $A E, A S$ & 102 & 1.31 & 53.50 \\
\hline
\end{tabular}


Table 2 Up-regulated miRNAs $(n=50)$ reported in at least two expression profiling studies (Continued)

\begin{tabular}{|c|c|c|c|c|}
\hline hsa-miR-93 & $A E, A S$ & 98 & 1.83 & 21.50 \\
\hline hsa-miR-125b-1 & $E J, M B$ & 80 & 12.62 & 16.50 \\
\hline hsa-miR-106b & $A E, N B$ & 61 & 1.33 & 36.00 \\
\hline hsa-miR-27a & $A E, N B$ & 49 & 2.70 & 22.00 \\
\hline hsa-miR-17 & $A P, S A$ & 42 & 2.77 & 14.50 \\
\hline hsa-miR-125b & $A E, A S$ & 25 & 1.89 & 22.00 \\
\hline
\end{tabular}

model was used to adjust for competing risk factors, and the hazard ratio (HR) with a $95 \%$ confidence interval (CI) was reported as an estimate of overall survival risk. The variables that were found to be significant in univariate analysis at $p<0.05$ were included in the final multivariate analysis in a backwards stepwise fashion. The statistical analyses were performed using the SPSS 18.0 for Windows software package (SPSS Inc.). Differences were considered to be statistically significant when the $p$-value was $<0.05$.

\section{Results}

Five hundred and ninety-eight relevant publications were indexed in the databases mentioned above (Scopus, GEO, PubMed and ArrayExpress). According to the inclusion criteria and the identification of duplicate

Table 3 Down-regulated miRNAs $(n=27)$ reported in at least two expression profiling studies

\begin{tabular}{|c|c|c|c|c|}
\hline miRNA name & Studies with same direction (reference) & No. of tissue samples tested & Mean fold-change & Mean rank \\
\hline hsa-miR-217 & $A E, A S, N A, N B, Y N$ & 371 & 18.16 & 4.20 \\
\hline hsa-miR-148a & $A E, A S, M B, N A, N B$ & 371 & 8.03 & 7.00 \\
\hline hsa-miR-375 & $A E, A S, M B, N A, N B$ & 371 & 4.86 & 9.40 \\
\hline hsa-miR-216b & $A S, N A, N B, Y N$ & 363 & 53.44 & 6.25 \\
\hline hsa-miR-216a & $A S, N A, N B, Y N$ & 363 & 30.17 & 2.25 \\
\hline hsa-miR-130b & $A E, A S, N A, N B$ & 310 & 6.17 & 12.25 \\
\hline hsa-miR-141 & $N B, S Z, A E, A S$ & 170 & 2.81 & 15.25 \\
\hline hsa-miR-30a-3p & $N A, N B, A E$ & 212 & 2.71 & 30.67 \\
\hline hsa-miR-200c & $A E, A S, N B$ & 150 & 2.66 & 23.67 \\
\hline hsa-miR-30a-5p & $A S, N B, A E$ & 150 & 2.16 & 27.67 \\
\hline hsa-miR-29c & $A E, A S, N B$ & 150 & 1.94 & 27.33 \\
\hline hsa-miR-30d & $A E, A S, N B$ & 150 & 1.73 & 35.33 \\
\hline hsa-miR-30e & $A S, N B, A E$ & 150 & 1.57 & 38.30 \\
\hline hsa-miR-379 & $\mathrm{SZ}, \mathrm{AE}, \mathrm{AS}$ & 122 & 1.62 & 21.67 \\
\hline has-miR-193b-3p & $N A, N B$ & 208 & 6.67 & 20.50 \\
\hline hsa-miR-184 & AS, YN & 159 & 2.82 & 26.50 \\
\hline hsa-miR-338-5p & $A S, N B$ & 142 & 3.15 & 25.50 \\
\hline hsa-miR-182 & $A E, A S$ & 102 & 2.88 & 15.50 \\
\hline hsa-miR-30b & $A E, A S$ & 102 & 2.25 & 17.00 \\
\hline hsa-miR-335 & $A E, A S$ & 102 & 2.16 & 15.00 \\
\hline hsa-miR-200a & $A E, A S$ & 102 & 1.66 & 24.50 \\
\hline hsa-miR-200b & $A E, A S$ & 102 & 1.62 & 28.00 \\
\hline hsa-miR-30c & AS, AS & 98 & 2.18 & 17.00 \\
\hline hsa-miR-148b & $A E, M B$ & 73 & 2.52 & 2.50 \\
\hline hsa-let-7f & $A E, S A$ & 37 & 13.05 & 20.00 \\
\hline hsa-let-7c & $A E, S A$ & 37 & 2.66 & 23.50 \\
\hline hsa-let-7b & $A E, S A$ & 37 & 1.97 & 25.00 \\
\hline
\end{tabular}


Table 4 Differentially expressed miRNAs $(n=21)$ with an inconsistent direction between two studies

\begin{tabular}{|c|c|c|c|c|c|}
\hline miRNA name & Direction of expression & $\begin{array}{c}\text { Studies with same direction } \\
\text { (reference) }\end{array}$ & $\begin{array}{c}\text { No. of tissue samples } \\
\text { tested }\end{array}$ & Mean fold-change & Mean rank \\
\hline \multirow[t]{2}{*}{ hsa-miR-103 } & $\uparrow$ & $A E, A P, A S, N B$ & 167 & 2.72 & 87.00 \\
\hline & $\downarrow$ & SZ & 20 & 1.73 & 5.00 \\
\hline \multirow[t]{2}{*}{ hsa-let-7d } & $\uparrow$ & $E J, A P$ & 32 & 6.82 & 11.50 \\
\hline & $\downarrow$ & $S A, A E$ & 37 & 7.04 & 22.50 \\
\hline \multirow[t]{2}{*}{ hsa-miR-26a } & $\uparrow$ & AP & 17 & 5.16 & 12.00 \\
\hline & $\downarrow$ & $A E, A S, S A$ & 131 & 4.38 & 30.67 \\
\hline \multirow[t]{2}{*}{ hsa-miR-146a } & $\uparrow$ & $A E, A S$ & 102 & 2.08 & 12.00 \\
\hline & $\downarrow$ & SA & 29 & 3.03 & 9.00 \\
\hline \multirow[t]{2}{*}{ hsa-miR-708 } & $\uparrow$ & $\mathrm{AS}, \mathrm{NA}$ & 254 & 3.15 & 43.50 \\
\hline & $\downarrow$ & NB & 48 & 9.26 & 7.00 \\
\hline \multirow[t]{2}{*}{ hsa-miR-345 } & $\uparrow$ & AS & 94 & 1.45 & 85.00 \\
\hline & $\downarrow$ & $E J, N B$ & 63 & 12.59 & 2.50 \\
\hline \multirow[t]{2}{*}{ hsa-miR-376a } & $\uparrow$ & EJ & 15 & 7.79 & 17.00 \\
\hline & $\downarrow$ & $A E, A S$ & 102 & 1.43 & 28.00 \\
\hline \multirow[t]{2}{*}{ hsa-miR-494 } & $\uparrow$ & NA & 160 & 4.23 & 41.00 \\
\hline & $\downarrow$ & $\mathrm{NB}, \mathrm{AE}$ & 56 & 3.86 & 14.50 \\
\hline \multirow[t]{2}{*}{ hsa-miR-423-5p } & $\uparrow$ & SA & 29 & 9.03 & 4.00 \\
\hline & $\downarrow$ & $\mathrm{YN}, \mathrm{NB}$ & 113 & 2.77 & 30.00 \\
\hline \multirow[t]{2}{*}{ hsa-miR-365 } & $\uparrow$ & SZ & 20 & 1.75 & 2.00 \\
\hline & $\downarrow$ & $A E, A S$ & 102 & 1.80 & 17.00 \\
\hline \multirow[t]{2}{*}{ hsa-miR-130a } & $\uparrow$ & NB & 48 & 2.00 & 28.00 \\
\hline & $\downarrow$ & $A E, A S$ & 102 & 1.62 & 29.50 \\
\hline \multirow[t]{2}{*}{ hsa-miR-132 } & $\uparrow$ & AS & 94 & 2.59 & 18.00 \\
\hline & $\downarrow$ & SZ & 20 & 3.05 & 1.00 \\
\hline \multirow[t]{2}{*}{ hsa-miR-324-3p } & $\uparrow$ & AS & 94 & 1.95 & 39.00 \\
\hline & $\downarrow$ & NB & 48 & 2.16 & 50.00 \\
\hline \multirow[t]{2}{*}{ hsa-miR-501-5p } & $\uparrow$ & AS & 94 & 1.59 & 64.00 \\
\hline & $\downarrow$ & NB & 48 & 2.02 & 52.00 \\
\hline \multirow[t]{2}{*}{ hsa-miR-874 } & $\uparrow$ & AS & 94 & 1.49 & 80.00 \\
\hline & $\downarrow$ & NB & 48 & 2.20 & 47.00 \\
\hline \multirow[t]{2}{*}{ hsa-miR-518d-3p } & $\uparrow$ & AS & 94 & 1.30 & 103.00 \\
\hline & $\downarrow$ & NA & 160 & 15.35 & 9.00 \\
\hline \multirow[t]{2}{*}{ hsa-miR-28-3p } & $\uparrow$ & AS & 94 & 1.28 & 104.00 \\
\hline & $\downarrow$ & NB & 48 & 4.49 & 23.00 \\
\hline \multirow[t]{2}{*}{ hsa-miR-648 } & $\uparrow$ & NA & 160 & 8.63 & 16.00 \\
\hline & $\downarrow$ & NB & 48 & 9.07 & 8.00 \\
\hline \multirow[t]{2}{*}{ hsa-miR-575 } & $\uparrow$ & NA & 160 & 7.52 & 22.00 \\
\hline & $\downarrow$ & NB & 48 & 4.38 & 24.00 \\
\hline \multirow[t]{2}{*}{ hsa-miR-877 } & $\uparrow$ & NA & 160 & 4.03 & 43.00 \\
\hline & $\downarrow$ & NB & 48 & 3.48 & 28.00 \\
\hline \multirow[t]{2}{*}{ hsa-let-7g } & $\uparrow$ & NB & 48 & 2.44 & 21.00 \\
\hline & $\downarrow$ & $A E$ & 8 & 1.06 & 45.00 \\
\hline
\end{tabular}




\begin{tabular}{|c|c|c|c|}
\hline miRNA name & No. of studies & Mean fold-change & Mean rank \\
\hline \multicolumn{4}{|l|}{ Up-regulated } \\
\hline hsa-miR-155 & 8 & 4.98 & 12.62 \\
\hline hsa-miR-21 & 7 & 2.95 & 12.29 \\
\hline hsa-miR-100 & 7 & 8.07 & 13.00 \\
\hline hsa-miR-221 & 7 & 6.71 & 11.42 \\
\hline hsa-miR-31 & 5 & 5.44 & 10.00 \\
\hline hsa-miR-10a & 5 & 2.50 & 14.60 \\
\hline hsa-miR-23a & 5 & 3.46 & 22.60 \\
\hline hsa-miR-143 & 5 & 4.03 & 9.40 \\
\hline hsa-miR-222 & 5 & 2.77 & 11.20 \\
\hline \multicolumn{4}{|l|}{ Down-regulated } \\
\hline hsa-miR-217 & 5 & 18.16 & 4.20 \\
\hline hsa-miR-148a & 5 & 8.03 & 7.00 \\
\hline hsa-miR-375 & 5 & 4.86 & 9.40 \\
\hline
\end{tabular}

publications, only fourteen independent studies [18-31] were included. However, one article was excluded for the unavailability of a ranked gene list both publically and in response to a request from the corresponding author [18]. The selection process is shown in Figure 1. Among the analysed studies, some of the studies employed patient samples as low as 5 [19] or 3 [20], which was too small to provide any reliable data. Not surprisingly, these two studies $[19,20]$ were the basis for excluding numerous candidates that were consistently reported as either up- or down-regulated in other studies. The most glaring example of the strategic error of including these two studies in our meta-analysis is miRNA-100, which, despite being reported to be up-

Table 6 PDAC meta-signature from the Robust Rank Aggregation method

\begin{tabular}{llll}
\hline miRNA name & $\begin{array}{l}\text { Corrected } \\
\boldsymbol{p} \text {-value }\end{array}$ & $\begin{array}{l}\text { Permutation } \\
\boldsymbol{p} \text {-value }\end{array}$ & No. of studies \\
\hline $\begin{array}{l}\text { Up-regulated } \\
\text { hsa-miR-155 }\end{array}$ & $6.17 \mathrm{E}-11$ & $8.64 \mathrm{E}-13$ & 8 \\
hsa-miR-100 & $3.32 \mathrm{E}-09$ & $7.01 \mathrm{E}-11$ & 7 \\
hsa-miR-21 & $2.75 \mathrm{E}-09$ & $3.29 \mathrm{E}-11$ & 7 \\
hsa-miR-221 & $1.56 \mathrm{E}-08$ & $9.34 \mathrm{E}-10$ & 7 \\
hsa-miR-31 & $1.44 \mathrm{E}-05$ & $8.83 \mathrm{E}-07$ & 5 \\
hsa-miR-143 & $6.78 \mathrm{E}-04$ & $4.56 \mathrm{E}-06$ & 5 \\
hsa-miR-23a & $3.27 \mathrm{E}-03$ & $5.09 \mathrm{E}-05$ & 5 \\
Down-regulated & & & 5 \\
hsa-miR-217 & $7.56 \mathrm{E}-07$ & $4.37 \mathrm{E}-09$ & 5 \\
hsa-miR-148a & $2.00 \mathrm{E}-05$ & $3.55 \mathrm{E}-07$ & 5 \\
hsa-miR-375 & $1.08 \mathrm{E}-03$ & $8.70 \mathrm{E}-06$ & 5 \\
\hline
\end{tabular}

regulated in 7 studies $[21,23,24,26,28,29,31]$, was considered to be down-regulated in one of the aforementioned studies [19], which only employed 5 tumour samples. Therefore, if Ref 19 was included, miR-100 would be listed as a miRNA with an inconsistent direction and would be subsequently excluded from the list of most consistently reported miRNAs. In addition, the fold-change in this study [19] was very low (less than 2) and may not have been significant if a large sample size was analysed. Other examples include miR-145, miR141, miR-379, miR-200c, and miR-125b, which were reported in an opposite direction solely in these two studies. To avoid these deviations, these two smallsample-size studies were excluded from our metaanalysis. A brief description of the eleven included studies [21-31] and the acronyms by which the studies are referred to in the following text are provided in Table 1.

The number of patients with PDAC that were investigated in these eleven studies ranged from 8 to 160 (median 47). The studies employed a diversity of microarray platforms (either commercial or custom), and the average number of miRNAs assayed was 778 (ranging from 377 to 1200; data were missing in three papers $[23,24,29]$ ). Only five studies [21-23,26,27] provided the whole list of differentially expressed miRNAs, while the others presented only a portion of their data. Our pooled dataset included a total of 538 tumour samples and 206 noncancerous control samples (at least), as in some studies, the number of noncancerous control samples was not specified [22].

A total of 439 differentially expressed miRNAs were reported in the eleven miRNA expression profiling studies; 254 were up-regulated and 185 were down-regulated in at least one study. Among the 439 miRNAs, 98 were reported in at least two studies; 77 (78.57\%) with a consistent direction (Tables 2 and 3 ) and 21 with an inconsistent direction (Table 4) among the studies. Among the 77 miRNAs with a consistent direction, 50 were reported to be up-regulated (Table 2) and 27 were reported to be down-regulated (Table 3). One miRNA (miR-155) was reported in eight studies, three miRNAs (miR-21, miR-100 and miR-221) were reported in seven studies and twelve miRNAs were reported in at least five studies, with a consistent direction in all reports (Table 5). The miRNAs that were consistently reported in at least five studies are shown in Table 5. Although there were no strong disagreements between the individual miRNA profiling studies, the top lists varied considerably from study to study.

Using the Robust Rank Aggregation method, we identified a statistically significant meta-signature of 7 up- and 3 down-regulated miRNAs in PDAC samples compared to noncancerous pancreatic tissues (Table 6). All meta-signature miRNAs that reached statistical significance after Bonferroni correction were reported 
Table 7 The ten most strongly enriched GO processes and pathways with respect to the meta-signature miRNA candidates

\begin{tabular}{|c|c|c|c|}
\hline \multicolumn{4}{|l|}{ GO processes } \\
\hline & Process & Hyp* & Genes \\
\hline & $\begin{array}{l}\text { 0006355: regulation of } \\
\text { transcription, DNA-dependent }\end{array}$ & $1.94 \mathrm{E}-31$ & 128 \\
\hline & $\begin{array}{l}\text { 0045944: positive regulation of } \\
\text { transcription from RNA } \\
\text { polymerase II promoter }\end{array}$ & $2.21 \mathrm{E}-18$ & 73 \\
\hline & $\begin{array}{l}\text { 0045893: positive regulation of } \\
\text { transcription, DNA-dependent }\end{array}$ & 7.64E-14 & 89 \\
\hline & $\begin{array}{l}\text { 0007275: multicellular organismal } \\
\text { development }\end{array}$ & 1.99E-13 & 57 \\
\hline & 0007165: signal transduction & $1.16 \mathrm{E}-10$ & 69 \\
\hline & $\begin{array}{l}\text { 0007399: nervous system } \\
\text { development }\end{array}$ & $8.52 \mathrm{E}-10$ & 74 \\
\hline & 0006915: apoptotic process & $1.76 \mathrm{E}-09$ & 57 \\
\hline & $\begin{array}{l}\text { 0045892: negative regulation of } \\
\text { transcription, DNA-dependent }\end{array}$ & 4.03E-09 & 55 \\
\hline & 0007155: cell adhesion & $5.06 \mathrm{E}-08$ & 90 \\
\hline & 0007411: axon guidance & 9.83E-08 & 24 \\
\hline \multicolumn{4}{|l|}{ KEGG Pathways } \\
\hline & Pathway & Hyp* & Genes \\
\hline & 05200: Pathways in cancer & $1.84 \mathrm{E}-05$ & 33 \\
\hline & 04010: MAPK signalling pathway & $3.62 \mathrm{E}-05$ & 31 \\
\hline & 04144: Endocytosis & $1.89 \mathrm{E}-04$ & 19 \\
\hline & 04510: Focal adhesion & 2.34E-04 & 25 \\
\hline & $\begin{array}{l}\text { 04810: Regulation of actin } \\
\text { cytoskeleton }\end{array}$ & 4.11E-04 & 22 \\
\hline & $\begin{array}{l}\text { 04350: TGF-beta signalling } \\
\text { pathway }\end{array}$ & 8.67E-04 & 12 \\
\hline & $\begin{array}{l}\text { 04141: Protein processing in } \\
\text { endoplasmic reticulum }\end{array}$ & 2.19E-03 & 18 \\
\hline & $\begin{array}{l}\text { 04630: Jak-STAT signalling } \\
\text { pathway }\end{array}$ & 5.07E-03 & 15 \\
\hline & 04310: Wnt signalling pathway & $5.29 \mathrm{E}-03$ & 14 \\
\hline & 04520: Adherens junction & $5.68 \mathrm{E}-03$ & 10 \\
\hline \multicolumn{4}{|c|}{ Panther pathways } \\
\hline & Pathway & Hyp* & Genes \\
\hline & P00057: Wnt signalling pathway & $6.66 \mathrm{E}-09$ & 36 \\
\hline & $\begin{array}{l}\text { P00012: Cadherin signalling } \\
\text { pathway }\end{array}$ & 8.93E-06 & 20 \\
\hline & $\begin{array}{l}\text { P00018: EGF receptor signalling } \\
\text { pathway }\end{array}$ & $1.25 \mathrm{E}-04$ & 18 \\
\hline & $\begin{array}{l}\text { P00034: Integrin signalling } \\
\text { pathway }\end{array}$ & 4.11E-04 & 17 \\
\hline & P00021: FGF signalling pathway & 8.83E-04 & 14 \\
\hline & P00047: PDGF signalling pathway & $2.18 \mathrm{E}-03$ & 13 \\
\hline & $\begin{array}{l}\text { P00060: Ubiquitin proteasome } \\
\text { pathway }\end{array}$ & 2.67E-03 & 11 \\
\hline & P00048: PI3 kinase pathway & $5.06 \mathrm{E}-03$ & 8 \\
\hline
\end{tabular}

Table 7 The ten most strongly enriched GO processes and pathways with respect to the meta-signature miRNA candidates (Continued)

\begin{tabular}{lll}
\hline $\begin{array}{l}\text { P00036: Interleukin signalling } \\
\text { pathway }\end{array}$ & $6.23 \mathrm{E}-03$ & 11 \\
& & \\
P04393: Ras pathway & $7.82 \mathrm{E}-03$ & 10 \\
\hline
\end{tabular}

The number of predicted target genes in the process or pathway is shown. Hyp*: corrected hypergeometric $p$-value.

by at least 5 datasets. Majority of the meta-signature miRNAs belong to the broadly conserved seed family (conserved across most vertebrates and bony fish).

Our results from the vote-counting strategy were almost the same with those from the Robust Rank Aggregation method. The ten GO processes and pathways that were most strongly enriched with respect to the metasignature miRNA candidates (miR-155, miR-100, miR-21, miR-221, miR-31, miR-143, miR-23a, miR-217, miR-148a and miR-375) are shown in Table 7.

\section{Experimental validation of the expression levels of the most deregulated miRNAs in patients with PDAC}

To determine if the ten most deregulated miRNAs from the meta-analysis (miR-155, miR-100, miR-21, miR-221, miR-31, miR-143, miR-23a, miR-217, miR-148a and miR375) could be used as diagnostic biomarkers of PDAC, the expression levels of these miRNAs were compared between PDAC tissues and neighbouring noncancerous tissues by qRT-PCR analysis. The results showed that the expression levels of miR-155, miR-100, miR-21, miR-221, miR-31, miR-143 and miR-23a were increased, whereas the levels of miR-217, miR-148a and miR-375 were decreased in the PDAC tissues (all $p<0.05$ ). Detailed data are available in Table 8.

\section{Determination of prognostic significance of the candidate miRNAs in PDAC}

The clinicopathological characteristics of 78 PDAC patients are shown in Table 9. The expression levels of individual miRNAs along with other well-known potential prognostic clinicopathological factors, such as histology, T category, lymph node metastasis, tumour size, perineural invasion, venous invasion and margin were included in a univariate analysis. With respect to the miRNA expression levels, for the up-regulated miRNAs, a fold-change of $\geq 2$ was defined as high expression, and a fold-change of $<2$ was defined as low expression; for the down-regulated miRNAs, a fold-change of $\geq 2$ was defined as low expression, and a fold-change of $<2$ was defined as high expression. Patients with advanced disease (UICC stage IV and concomitance of distant metastases) were excluded because we assumed that the prognosis of these patients $(n=8)$ is determined by the occurrence of relapse or metastasis rather than other 
Table 8 Relative expression of miRNAs in PDAC compared with matched normal pancreatic tissue controls determined by qRT-PCR

\begin{tabular}{ccccc}
\hline $\begin{array}{c}\text { miRNA name } \\
\text { Up-regulated }\end{array}$ & PDAC & $\mathbf{N}$ & $\boldsymbol{p}$-value & Fold-change \\
\hline miR-155 & $5.56 \pm 1.00$ & $2.71 \pm 0.66$ & $<0.001$ & $2.11 \pm 0.41$ \\
miR-100 & $7.40 \pm 2.21$ & $3.91 \pm 1.32$ & $<0.001$ & $2.00 \pm 0.51$ \\
miR-21 & $3.80 \pm 0.99$ & $1.7 \pm 0.35$ & $<0.001$ & $2.25 \pm 0.44$ \\
miR-221 & $8.03 \pm 2.77$ & $3.26 \pm 0.67$ & $<0.001$ & $2.53 \pm 0.84$ \\
miR-31 & $6.52 \pm 0.98$ & $2.93 \pm 0.39$ & $<0.001$ & $2.12 \pm 0.47$ \\
miR-143 & $7.45 \pm 1.22$ & $2.21 \pm 1.43$ & $<0.001$ & $2.94 \pm 0.74$ \\
miR-23a & $7.80 \pm 1.18$ & $3.44 \pm 0.73$ & $<0.001$ & $2.35 \pm 0.52$ \\
Down-regulated & & & & \\
miR-217 & $2.88 \pm 1.15$ & $10.35 \pm 3.68$ & $<0.001$ & $3.91 \pm 1.36$ \\
miR-148a & $3.85 \pm 1.48$ & $10.39 \pm 2.97$ & $<0.001$ & $2.86 \pm 0.77$ \\
miR-375 & $4.00 \pm 1.55$ & $7.05 \pm 1.99$ & $<0.001$ & $1.76 \pm 0.36$ \\
\hline
\end{tabular}

Data are expressed as the mean \pm SD. N: matched normal pancreatic tissue.

biological characteristics, such as miRNA expression levels.

Kaplan-Meier survival analysis was used to analyse the association between postoperative survival and the miRNA expression level, and the resulting curves were divided into two classes (high and low expression in comparison with the mean level of miRNA expression as the threshold), as shown in Figure 2.

A univariate analysis using the Cox hazard regression model demonstrated that a high expression level of miR-21 $(p=0.018, \mathrm{HR}=2.610 ; 95 \% \mathrm{CI}=1.179-5.777)$ and $\mathrm{miR}-155$ ( $p=0.035, \mathrm{HR}=2.414 ; 95 \% \mathrm{CI}=1.064-5.478)$, a low expression level of miR-375 ( $p=0.022, \mathrm{HR}=2.337 ; 95 \% \mathrm{CI}=1.431-5.066)$, $\mathrm{T}$ category $(p=0.039, \mathrm{HR}=2.282 ; 95 \% \mathrm{CI}=1.043-4.994)$ and margin involvement $(p=0.026, \mathrm{HR}=2.550$; $95 \% \mathrm{CI}=1.120$ $5.805)$ are associated with poor patient survival.

\section{Identification of two prognostic miRNAs by multivariate analysis}

In a multivariate analysis using the Cox hazard regression model, a high expression level of miR-21 ( $p=0.021$, $\mathrm{HR}=2.599 ; 95 \% \mathrm{CI}=1.151-5.867)$, a low expression level of miR-375 ( $p=0.034, \mathrm{HR}=2.451 ; 95 \% \mathrm{CI}=1.429-5.135)$ and margin involvement $(p=0.030, \mathrm{HR}=2.543$; $95 \% \mathrm{CI}=1.093$ 5.918 ) were identified as significant unfavourable prognostic factors (Table 10).

\section{Discussion}

The common drawback of miRNA expression profiling studies is the lack of agreement among several studies. Differences in measurement platforms and lab protocols as well as small sample sizes can render gene expression levels incomparable. Sato et al. [32] and Wang et al. [33] systematically analysed representative miRNA profiling
Table 9 Clinicopathological characteristics of 78 PDAC patients

\begin{tabular}{|c|c|}
\hline Gender & \\
\hline Male & $44(56 \%)$ \\
\hline Female & $34(44 \%)$ \\
\hline \multicolumn{2}{|l|}{ T category } \\
\hline T1 & $14(18 \%)$ \\
\hline $\mathrm{T} 2$ & $26(33 \%)$ \\
\hline T3 & $28(36 \%)$ \\
\hline T4 & $10(13 \%)$ \\
\hline \multicolumn{2}{|l|}{$\mathrm{N}$ category } \\
\hline NO & $34(44 \%)$ \\
\hline N1 & $44(56 \%)$ \\
\hline \multicolumn{2}{|l|}{ M category } \\
\hline MO & $70(90 \%)$ \\
\hline M1 & $8(10 \%)$ \\
\hline \multicolumn{2}{|l|}{ Tumour size } \\
\hline$\geq 2 \mathrm{~cm}$ & $42(54 \%)$ \\
\hline$<2 \mathrm{~cm}$ & $36(46 \%)$ \\
\hline \multicolumn{2}{|l|}{ Histology } \\
\hline Well or moderately differentiated & 38 (49\%) \\
\hline Poorly differentiated & $40(51 \%)$ \\
\hline \multicolumn{2}{|l|}{ Perineural invasion } \\
\hline None or slight & $46(59 \%)$ \\
\hline Prominent & $32(41 \%)$ \\
\hline \multicolumn{2}{|l|}{ Venous invasion } \\
\hline None or slight & $40(51 \%)$ \\
\hline Prominent & $38(49 \%)$ \\
\hline \multicolumn{2}{|l|}{ Tumour grade (UICC) } \\
\hline Stage I-IIA & $32(41 \%)$ \\
\hline Stage IIB-IV & 46 (59\%) \\
\hline \multicolumn{2}{|l|}{ Resection margin status } \\
\hline Ro & $32(41 \%)$ \\
\hline R1 & $46(59 \%)$ \\
\hline
\end{tabular}

platforms and revealed that each platform is relatively stable in terms of its own intra-reproducibility; however, the inter-platform reproducibility among different platforms is low. Although the ideal method involves the analysis the raw miRNA expression datasets that are pooled together, such a rigorous approach is often impossible due to the unavailability of raw data and the low inter-platform concordance of results among different studies would bring difficulties to the analysis. To overcome these limitations, it might be better to analyse datasets separately and then aggregate the resulting gene lists. In this study, we used a meta-analysis approach to analyse PDAC-specific miRNAs derived from independent 
profiling experiments. The well-known vote-counting strategy [12,13] and the recently published Robust Rank Aggregation method [16,17] were employed. The core elements of the two methods were searches for the most recognised miRNAs among the included studies.

Two principal methods are used to measure miRNA expression levels: qRT-PCR and microarray hybridisation. The technological merits and drawbacks of qRTPCR and microarrays for miRNA analysis are similar to those for RNA or genomic DNA quantification [34]. RT$\mathrm{PCR}$, a semiquantitative method, is labour intensive and provides data for only one, or very few, miRNA(s) per assay. However, the rapid increase in the number of known miRNAs renders this method inefficient on a genomic scale, and it is most likely better used as a tool for validation rather than discovery. Microarrays are the best option for a standardised genome-wide assay that is amenable to high-throughput application [35]. As qRTPCR detects only preselected miRNAs, mostly the miRNAs that were shown to be differentially expressed in PDAC from normal tissue in other studies, it hinders the discovery of new miRNAs. Most importantly, the results of studies using qRT-PCR analysis [36-40] were consistent with those of microarray-based studies. In addition to the intra-platform deviations between microarray and qRT-PCR analyses [35], we excluded qRT-PCR-based studies and focused on studies using miRNA microarray platforms.

We identified a meta-signature of seven up- and three down-regulated miRNAs. To our knowledge, no metaanalysis of miRNA profiling studies has specifically investigated PDAC. Furthermore, this is the first study that used a combination of the two most commonly used methods in the meta-analysis of miRNA and gene
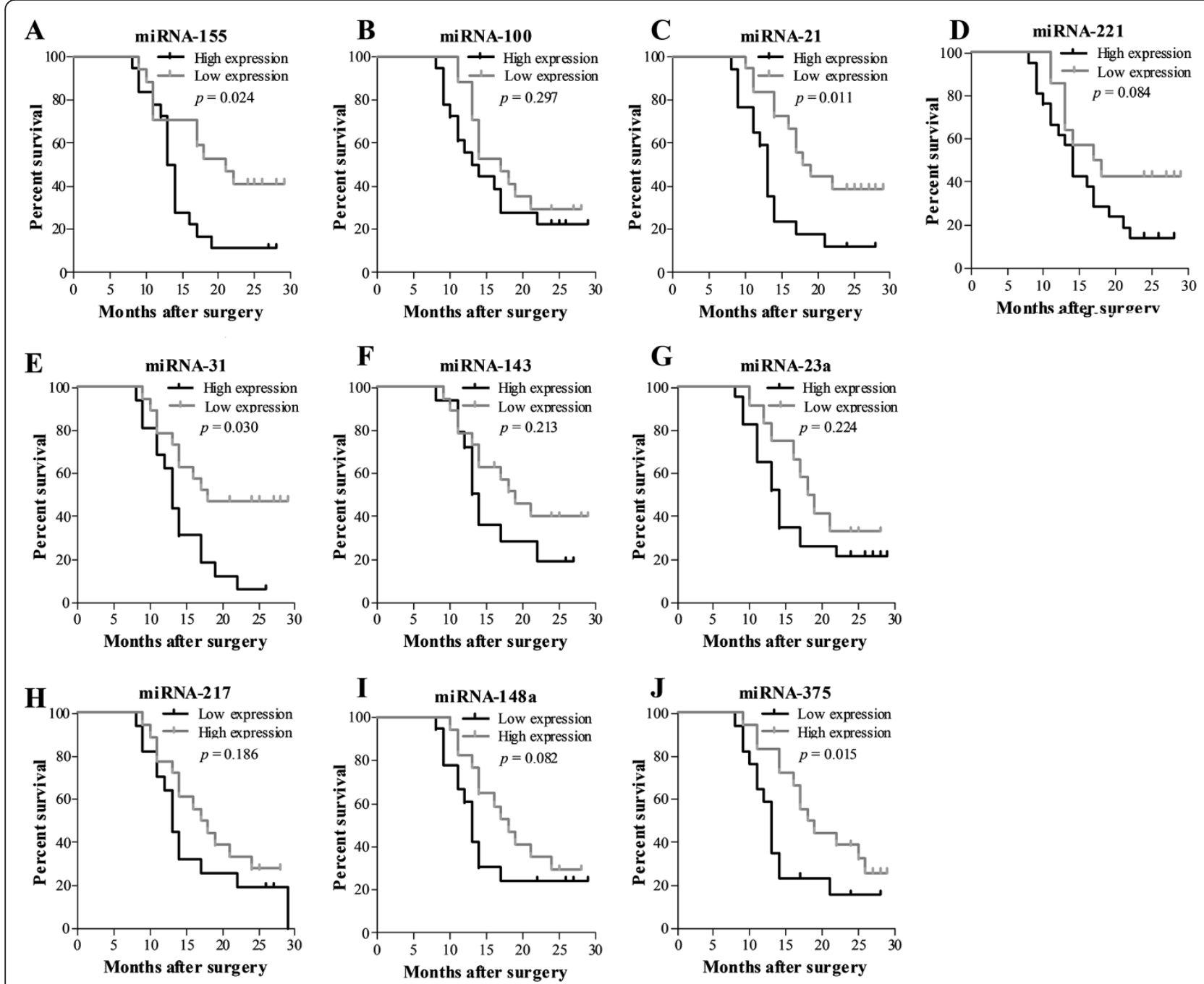

Figure 2 Kaplan-Meier analysis of overall survival in patients with PDAC based on their expression of miR-155 (A), miR-100 (B), miR-21 (C), miR-221 (D), miR-31 (E), miR-143 (F), miR-23a (G), miR-217 (H), miR-148a (I) and miR-375 (J). p-values are based on the log-rank test. 
Table 10 Univariate and multivariate survival analysis of the clinicopathological and molecular features of PDAC

\begin{tabular}{|c|c|c|c|c|c|}
\hline \multirow[t]{2}{*}{ Factor } & & \multicolumn{2}{|c|}{ Univariate analysis } & \multicolumn{2}{|c|}{ Multivariate analysis } \\
\hline & & HR $(95 \% \mathrm{Cl})$ & $p$-value & $\mathrm{HR}(95 \% \mathrm{Cl})$ & $p$-value \\
\hline Histology & Well or moderate vs. poor & $1.342(0.621-2.901)$ & 0.454 & & \\
\hline T category & T 1/2 VS. T $3 / 4$ & $2.282(1.043-4.994)$ & 0.039 & $1.518(0.666-3.460)$ & 0.320 \\
\hline Lymph node metastasis & Negative vs. positive & $1.935(0.867-4.317)$ & 0.107 & & \\
\hline Tumour size & $<2 \mathrm{~cm}$ vs. $\geq 2 \mathrm{~cm}$ & $1.736(0.790-3.814)$ & 0.170 & & \\
\hline Perineural invasion & None or slight vs. prominent & $1.244(0.563-2.752)$ & 0.589 & & \\
\hline Margin involvement & R0 vs. R1 & $2.550(1.120-5.805)$ & 0.026 & $2.543(1.093-5.918)$ & 0.030 \\
\hline Vascular invasion & None or slight vs. prominent & $2.542(1.154-5.601)$ & 0.021 & $1.940(0.819-4.597)$ & 0.132 \\
\hline miR-155 expression & High vs. low & $2.414(1.064-5.478)$ & 0.035 & $1.365(0.520-3.579)$ & 0.538 \\
\hline miR-100 expression & High vs. low & $1.480(0.683-3.205)$ & 0.321 & & \\
\hline miR-21 expression & High vs. low & $2.610(1.179-5.777)$ & 0.018 & $2.599(1.151-5.867)$ & 0.021 \\
\hline miR-221 expression & High vs. low & $2.001(0.868-4.617)$ & 0.104 & & \\
\hline miR-31 expression & High vs. low & $2.735(1.317-6.426)$ & 0.039 & $2.637(1.298-6.635)$ & 0.048 \\
\hline miR-143 expression & High vs. low & $1.516(1.211-4.429)$ & 0.257 & & \\
\hline miR-23a expression & High vs. low & 1.639 (0.709-3.788) & 0.248 & & \\
\hline miR-217 expression & Low vs. high & $1.419(1.045-4.021)$ & 0.205 & & \\
\hline miR-148a expression & Low vs. high & $1.739(1.385-4.481)$ & 0.093 & & \\
\hline miR-375 expression & Low vs. high & $2.337(1.431-5.066)$ & 0.022 & $2.451(1.429-5.135)$ & 0.034 \\
\hline
\end{tabular}

profiling. To determine if the identified miRNAs could be used as diagnostic biomarkers, we experimentally validated the expression of these miRNAs in a set of PDAC samples.

There are several factors that must be considered when choosing miRNAs as candidate diagnostic biomarkers for PDAC. First, the fold-change of the biomarker should be significant enough to discriminate cancerous tissue from benign tissue. As is shown in Tables 2 and 3, the average fold changes of the 10 miRNAs identified in the microarray-based studies were all $>2$. In addition, the candidate miRNAs should be expressed in a majority of tissues. As was validated by qRT-PCR, the upregulated miRNAs were all expressed in more than $85 \%$ of the samples tested (data not shown).

Second, the biological function of each individual miRNA should be thoroughly investigated. A single miRNA may have dozens of targets, and a specific mRNA may be regulated by multiple different miRNAs [7]. A better understanding of the targets of the miRNAs would advance their use in clinical settings. As shown in Table 7 , the ten most strongly enriched GO processes and pathways with respect to the meta-signature miRNA candidates were identified. In the GO processes list, regulation of transcription, DNA-dependent; positive regulation of transcription from RNA polymerase II promoter; and positive regulation of transcription, DNA-dependent were ranked as the top three, which is in accordance with the known primary functions of miRNAs [6,7]. Pathways in cancer and Wnt signalling pathways were ranked first in the KEGG and
Panther pathway lists, respectively, highlighting the essential roles of miRNAs in cancer development.

Third, there should be adequate information about the pattern of expression of the miRNAs in different types of specimens. It has been indicated that circulating miRNAs in plasma could be more tissue-specific than tumour-specific $[41,42]$. In the context of the vast inconsistency between tissue-based and plasma-based results [23], we focused on studies that analysed miRNA expression between PDAC tissues and noncancerous pancreatic tissues in humans.

Last but not least, rigorous validation and demonstration of reproducibility in an independent cohort of patients are necessary to confirm the diagnostic value of miRNAs. With this in mind, we experimentally validated 10 candidate miRNAs in PDAC samples and confirmed that these 10 miRNAs were differentially expressed between PDAC tissues and noncancerous pancreatic tissues.

Considering that miRNA expression is able to successfully discriminate normal from cancerous pancreatic tissues, it is tempting to speculate that miRNAs could also predict cancer prognosis. However, our results do not exclude the possibility that other miRNAs are associated with prognosis, as we only studied a meta-signature of 10 miRNAs in a limited number of PDAC samples $(\mathrm{n}=78)$. The main reason for the possible association between miRNAs not within this meta-signature and prognosis may centre on the relatively small sample size in our study and others [25,27]. It is quite unrealistic to include all the miRNAs in Kaplan-Meier survival analyses, 
as it would be very laborious and time-consuming. Thus, commonly, only the candidate miRNAs with the greatest fold changes are included. As mentioned above, although there were no strong disagreements between the individual miRNA profiling studies, the top lists varied considerably from study to study. To remedy this problem, it was critical to identify the most differentially expressed miRNAs. We used a meta-review approach, which combines the results of several individual studies to increase statistical power and to subsequently resolve the inconsistency among different profiling studies. A meta-signature of seven up- and three down-regulated miRNAs was identified. Then, in independent patient samples, miR-21, miR-31 and miR-375 were found to be associated with cancer prognosis.

From our point of view, great caution should be taken in future research in this field. To start, sample sizes should be increased to minimise random sampling error. Next, as it is impossible for every researcher to use the same platform, reliable microarray platforms should be employed in all experiments. Finally, it is advisable to obtain an integrated view of the candidate miRNAs from many studies to avoid one-sided opinions, as great discrepancies exist among the studies.

Our study presents a method to resolve the differences that exist among studies and might have some clinical significance for research on miRNAs in PDAC. The 10 identified miRNAs may be used as diagnostic biomarkers or even therapeutic targets. In addition to our metaanalysis, we performed further studies examining the expression of the candidate miRNAs in PDAC samples and confirmed miR-21, miR-31 and miR-375 as potential prognostic biomarkers for PDAC.

\section{Competing interests}

The authors declare that they have no competing interests.

\section{Authors' contributions}

MZM, XK and MZW conceived the study and participated in the data collection and analysis. MZM, XK and MZW performed the experiments. MZM and KX analysed the data. MZM, XK, ZWQ, WG and CHP wrote the paper. All authors read and approved the final manuscript.

\section{Acknowledgements \\ This work was supported by National Natural Science Foundation of China (grant no. 81272747). The funding sources had no role in the study design, data collection, analysis or interpretation, or the writing of this manuscript. The authors thank the Department of General Surgery of Ruijin Hospital for providing the PDAC tissue samples and Dr. Fei Yuan for the pathology assessments. \\ Author details \\ ${ }^{1}$ Department of General Surgery, Xinhua Hospital, Shanghai Jiaotong University School of Medicine, 1665 Kongjiang Road, Shanghai 200092, People's Republic of China. 'Department of Endocrinology, Xinhua Hospital, Shanghai Jiaotong University School of Medicine, Shanghai, China. ${ }^{3}$ Department of Pharmacology, Wannan Medical College, Anhui, China. ${ }^{4}$ Department of General Surgery, Ruijin Hospital, Shanghai Jiaotong University School of Medicine, Shanghai, China.}

Received: 14 May 2013 Accepted: 26 September 2013 Published: 28 September 2013

\section{References}

1. Hidalgo M: New insights into pancratic cancer biology. Ann Oncol 2012, 23(Suppl 10):135-138.

2. Hidalgo M: Pancreatic cancer. N Engl J Med 2010, 362:1605-1617.

3. Mardis ER: Applying next-generation sequencing to pancreatic cancer treatment. Nat Rev Gastroenterol Hepatol 2012, 9:477-486.

4. Du Y, Liu M, Gao J, Li Z: Aberrant microRNAs expression patterns in pancreatic cancer and their clinical translation. Cancer Biother Radiopharm 2013, 28:361-369.

5. Costello E, Greenhalf W, Neoptolemos JP: New biomarkers and targets in pancreatic cancer and their application to treatment. Nat Rev Gastroenterol Heaptol 2012, 9:435-444.

6. Gregory RI, Chendrimada TP, Cooch N, Shiekhattar R: Human RISC couples microRNA biogenesis and posttranscriptional gene silencing. Cell 2005, 123:631-640.

7. Yates LA, Norbury CJ, Gilbert RJ: The long and short of microRNAs. Cell 2013, 153:516-519.

8. Singh R, Mo YY: Role of microRNAs in breast cancer. Cancer Biol Ther 2013, 14:201-212

9. Baer C, Claus R, Plass C: Genome-wide epigenetic regulation of miRNAs in cancer. Cancer Res 2013, 73:473-477.

10. Song S, Ajani JA: The role of microRNAs in cancers of upper gastrointestinal tract. Nat Rev Gastroenterol Hepatol 2013, 10:109-118.

11. Lee HK, Hsu AK, Sajdak J, Qin J, Pavlidis P: Coexpression analysis of human genes across many microarray data sets. Genome Res 2004, 14:1085-1094.

12. Griffith OL, Melck A, Jones SJ, Wiseman SM: Meta-analysis and meta-review of thyroid cancer gene expression profiling studies identifies important diagnostic biomarkers. J Clin Oncol 2006, 24:5043-5051.

13. Chan SK, Griffith OL, Tai IT, Jones SJ: Meta-analysis of colorectal cancer gene expression profiling studies identifies consistently reported candidate biomarkers. Cancer Epidemiol Biomarkers Prev 2008, 17:543-552.

14. Ma Y, Zhang P, Yang J, Liu Z, Yang Z, Qin H: Candidate microRNA biomarkers in human colorectal cancer: systematic review profiling studies and experimental validation. Int J Cancer 2012, 130:2077-2087.

15. Guan P, Yin Z, Li X, Wu W, Zhou B: Meta-analysis of human lung cancer microRNA expression profiling studies comparing cancer tissues with normal tissues. J Exp Clin Cancer Res 2012, 31:54.

16. Kolde R, Laur $S$, Adler P, Vilo J: Robust rank aggregation for gene list integration and meta-analysis. Bioinformatics 2012, 28:573-580.

17. Võsa U, Vooder T, Kolde R, Vilo J, Metspalu A, Annilo T: Meta-analysis of microRNA expression in lung cancer. Int J Cancer 2013, 132:2884-2893.

18. Singh S, Chitkara D, Kumar V, Behrman SW: Mahato RI:miRNA profiling in pancreatic cancer and restoration of chemosensitivity. Cancer Lett 2012, 12:00596-4.

19. Munding JB, Adai AT, Maghnouj A, Urbanik A, Zöllner H, Liffers ST, Chromik AM, Uhl W, Szafranska-Schwarzbach AE, Tannapfel A, Hahn SA: Global microRNA expression profiling of microdissected tissues identifies miR$135 \mathrm{~b}$ as a novel biomarker for pancreatic ductal adenocarcinoma. Int $J$ Cancer 2012, 131:E86-E95.

20. Ma Y, Yu S, Zhao W, Lu Z, Chen J: miR-27a regulates the growth, colony formation and migration of pancreatic cancer cells by targeting Sprouty2. Cancer Lett 2010, 298:150-158.

21. Szafranska AE, Davison TS, John J, Cannon T, Sipos B, Maghnouj A, Labourie E, Hahn SA: MicroRNA expression alterations are linked to tumorigenesis and non-neoplastic processes in pancreatic ductal adenocarcinoma. Oncogene 2007, 26:4442-4452.

22. Piepoli A, Tavano F, Copetti M, Mazza T, Palumbo O, Panza A, di Mola FF, Pazienza V, Mazzoccoli G, Biscaglia G, Gentile A, Mastrodonato N, Carella M, Pellegrini F, di Sebastiano P, Andriulli A: Mirna expression profiles identify drivers in colorectal and pancreatic cancers. PLoS One 2012, 7:e33663.

23. Bauer AS, Keller A, Costello E, Greenhalf W, Bier M, Borries A, Beier M, Neoptolemos J, Büchler M, Werner J, Giese N, Hoheisel JD: Diagnosis of pancreatic ductal adenocarcinoma and chronic pancreatitis by measurement of microRNA abundance in blood and tissue. PLoS One 2012, 7:e34151.

24. Lee EJ, Gusev Y, Jiang J, Nuovo GJ, Lerner MR, Frankel WL, Morgan DL, Postier RG, Brackett DJ, Schmittgen TD: Expression profiling identifies microRNA signature in pancreatic cancer. Int J Cancer 2007, 120:1046-1054.

25. Bloomston M, Frankel WL, Petrocca F, Volinia S, Alder H, Hagan JP, Liu CG, Bhatt D, Taccioli C, Croce CM: MicroRNA expression patterns to differentiate pancreatic adenocarcinoma from normal pancreas and chronic pancreatitis. JAMA 2007, 297:1901-1908. 
26. Schultz NA, Werner J, Willenbrock H, Roslind A, Giese N, Horn T, Wøjdemann M, Johansen JS: MicroRNA expression profiles associated with pancreatic adenocarcinoma and ampullary adenocarcinoma. Mod Pathol 2012, 25:1609-1622.

27. Jamieson NB, Morran DC, Morton JP, Ali A, Dickson EJ, Carter CR, Sansom OJ, Evans TR, McKay CJ, Oien KA: MicroRNA molecular profiles associated with diagnosis, clinicopathologic criteria, and overall survival in patients with resectable pancreatic ductal adenocarcinoma. Clin Cancer Res 2012, 18:534-545.

28. Panarelli NC, Chen YT, Zhou XK, Kitabayashi N, Yantiss RK: MicroRNA expression aids the preoperative diagnosis of pancreatic ductal adenocarcinoma. Pancreas 2012, 41:685-690.

29. Ali S, Saleh H, Sethi S, Sarkar FH, Philip PA: MicroRNA profiling of diagnostic needle aspirates from patients with pancreatic cancer. Br J Cancer 2012, 107:1354-1360.

30. Zhang S, Hao J, Xie F, Hu X, Liu C, Tong J, Zhou J, Wu J, Shao C: Downregulation of miR-132 by promoter methylation contributes to pancreatic cancer development. Carcinogenesis 2011, 32:1183-1189.

31. Nagao Y, Hisaoka M, Matsuyama A, Kanemitsu S, Hamada T, Fukuyama T, Nakano R, Uchiyama A, Kawamoto M, Yamaguchi K, Hashimoto H: Association of microRNA-21 expression with its targets, PDCD4 and TIMP3, in pancreatic ductal adenocarcinoma. Mod Pathol 2012, 25:112-121.

32. Sato F, Tsuchiya S, Terasawa L, Tsujimoto G: Intra-platform repeatability and inter-platform comparability of microRNA microarray technology. Plos One 2009, 4:e5540.

33. Wang B, Howel P, Bruheim S, Ju J, Owen LB, Fodstad O, Xi Y: Systematic evaluation of three microRNA profiling platforms: microarray, breads array, and quantative real-time PCR array. Plos One 2011, 6:e17167.

34. Git A, Dvinge H, Salmon-Divon M, Osborne M, Kutter C, Hadfield J, Bertone $P$, Caldas C: Systematic comparison of microarray profiling, real-time PCR, and next-generation sequencing technologies for measuring differential microRNA expression. RNA 2010, 16:991-1006.

35. Etienne W, Meyer MH, Peppers J, Meyer RA Jr: Comparison of mRNA gene expression by RT-PCR and DNA microarray. Biotechniques 2004, 36:618-620

36. Zhang XJ, Ye H, Zeng CW, He B, Zhang H, Chen YQ: Dysregulation of miR15a and miR-214 in human pancreatic cancer. J Hematol Oncol 2010, 3:46.

37. Tavano F, di Mola FF, Piepoli A, Panza A, Copetti M, Burbaci FP, Latiano T, Pellegrini F, Maiello E, Andriulli A, di Sebastiano P: Changes in miR-143 and miR-21 expression and clinicopathological correlations in pancreatic cancers. Pancreas 2012, 41:1280-1284.

38. Greither $T$, Grochola LF, Udelnow A, Lautenschlager C, Wurl P, Taubert $H$ : Elevated expression of microRNAs 155, 203, 210 and 222 in pancreatic tumors is associated with poorer survival. Int I Cancer 2010, 126:73-80.

39. Papaconstantinou IG, Manta A, Gazouli M, Lyberopoulou A, Lykou dis PM Polymeneas G, Voros D: Expression of microRNAs in patients with pancreatic cancer and its prognostic significance. Pancreas 2013, 42:67-71.

40. Ikenaga N, Ohuchida K, Mizumoto K, Yu J, Kayashima T, Sakai H, Fujita H, Nakata K, Tanaka M: MicroRNA-203 expression as a new prognostic marker of pancreatic adenocarcinoma. Ann Surg Oncol 2010, 17:3210-3218.

41. Liu CG, Calin GA, Meloon B, Gamliel N, Sevignani C, Ferracin M, Dumitru CD, Shimizu M, Zupo S, Dono M, Alder H, Bullrich F, Negrini M, Croce CM: An oligonucleotide microchip for genome-wide microRNA profiling in human and mouse tissues. Proc Natl Acad Sci USA 2004, 101:9740-9744.

42. Babak T, Zhang W, Morris Q, Blencowe BJ, Hughes TR: Probing microRNAs with microarrays: tissue specificity and functional inference. RNA 2004, 10:1813-1819

doi:10.1186/1756-9966-32-71

Cite this article as: Ma et al: Candidate microRNA biomarkers of pancreatic ductal adenocarcinoma: meta-analysis, experimental validation and clinical significance. Journal of Experimental \& Clinical Cancer Research 2013 32:71.

\section{Submit your next manuscript to BioMed Central and take full advantage of:}

- Convenient online submission

- Thorough peer review

- No space constraints or color figure charges

- Immediate publication on acceptance

- Inclusion in PubMed, CAS, Scopus and Google Scholar

- Research which is freely available for redistribution

Submit your manuscript at www.biomedcentral.com/submit
C Biomed Central 